\title{
The semantic category-based grouping in the Multiple Identity Tracking task
}

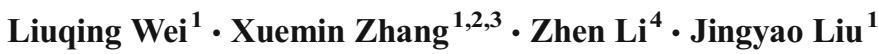

Published online: 27 September 2017

(C) The Psychonomic Society, Inc. 2017

\begin{abstract}
In the Multiple Identity Tracking (MIT) task, categorical distinctions between targets and distractors have been found to facilitate tracking (Wei, Zhang, Lyu, \& Li in Frontiers in Psychology, 7, 589, 2016). The purpose of this study was to further investigate the reasons for the facilitation effect, through six experiments. The results of Experiments 13 excluded the potential explanations of visual distinctiveness, attentional distribution strategy, and a working memory mechanism, respectively. When objects' visual information was preserved and categorical information was removed, the facilitation effect disappeared, suggesting that the visual distinctiveness between targets and distractors was not the main reason for the facilitation effect. Moreover, the facilitation effect was not the result of strategically shifting the attentional distribution, because the targets received more attention than the distractors in all conditions. Additionally, the facilitation effect did not come about because the identities of targets were encoded and stored in visual working memory to assist in the recovery from tracking errors; when working memory was disturbed by the object identities changing during
\end{abstract}

Xuemin Zhang

xmzhang@bnu.edu.cn

1 Beijing Key Laboratory of Applied Experimental Psychology, National Demonstration Center for Experimental Psychology Education (Beijing Normal University), Faculty of Psychology, Beijing Normal University, Beijing, China

2 State Key Laboratory of Cognitive Neuroscience and Learning and IDG/McGovern Institute for Brain Research, Beijing Normal University, Beijing, China

3 Center for Collaboration and Innovation in Brain and Learning Sciences, Beijing Normal University, Beijing, China

4 eMetric, LLC, San Antonio, TX, USA tracking, the facilitation effect still existed. Experiments 4 and 5 showed that observers grouped targets together and segregated them from distractors on the basis of their categorical information. By doing this, observers could largely avoid distractor interference with tracking and improve tracking performance. Finally, Experiment 6 indicated that category-based grouping is not an automatic, but a goal-directed and effortful, strategy. In summary, the present findings show that a semantic category-based target-grouping mechanism exists in the MIT task, which is likely to be the major reason for the tracking facilitation effect.

Keywords Categorization · Grouping · Segmentation . Working memory

The Multiple Identity Tracking (MIT) task (Oksama \& Hyönä, 2004, 2008), derived from the Multiple Object Tracking (MOT) task (Pylyshyn \& Storm, 1988), has been used to study the effect of surface features on attentive tracking or the binding of objects' identities and locations during multiple objects' motion. In a variant of the MIT task, observers only need to track the targets' locations among the distractors, without specifying their identities. Makovski and Jiang (2009b) found that location-tracking performance was enhanced when the objects were unique in color. Specifically, observers' performance at tracking four targets among eight uniquely colored objects (four distractors) was better than that of tracking four identical objects. Moreover, tracking uniquely colored objects interfered with a concurrent color memory task, which indicated that attentive tracking and visual working memory share common cognitive resources (Fougnie \& Marois, 2006). The findings of Makovski and Jiang (2009b) demonstrated that surface properties are stored in visual working memory, allowing observers to recover from errors during 
tracking and enhance the functional resolution of attention. In another study, Makovski and Jiang (2009a) compared observers' tracking performance in unique-object conditions with that in homogeneous conditions. The enhancement by distinctive features was retained in conditions under which at least a single feature differentiated each object has one unique feature. For instance, attentive tracking was more accurate when all of the objects were unique in color or digit identity than when they were identical in color and digit identity. However, the enhancement disappeared when the targets' colors or digit identities were shared by the distractors, even if all objects were distinct from one another in the conjunction of color and digits. Feria (2012) demonstrated that distractors with distinct features (shape, color, or motion) from the targets impaired tracking less than did distractors identical to the targets, and she suggested that observers could use the distinct surface properties between targets and distractors to facilitate tracking.

However, Ren, Chen, Liu, and Fu (2009) used human faces as tracking stimuli and found that unique faces impaired tracking as compared with identical faces. Liu, Chen, Liu, and Fu (2012), in their later study, investigated why objects' uniqueness produced the opposite effects on tracking performance shown by previous research (Makovski \& Jiang, 2009a, b; Ren et al., 2009). They revealed that whether objects' uniqueness would enhance or impair tracking primarily depended on the objects' visual complexity: When object stimuli were less complex, such as numbers of one- or two-digit length or simple Chinese characters, the unique identities enhanced tracking performance as compared with homogeneous condition. When object stimuli were visually complex, such as numbers of three- or four-digit length or complex Chinese characters, the objects' uniqueness impaired tracking performance. One explanation is that visually complex objects had a lower working memory span, so that processing of the objects' identities consumed extra cognitive resources and interfered with attentive tracking.

Early studies had mainly examined the effect of surface property uniqueness on attentive tracking. For example, when objects had different surface features or identities, to what degree would attentive tracking of the objects' motion be influenced? However, researchers have recently found that automatic feature-based grouping exists in the MIT task (Erlikhman, Keane, Mettler, Horowitz, \& Kellman, 2013; Keane, Mettler, Tsoi, \& Kellman, 2011; Wang, Zhang, Li, \& Lyu, 2016). The grouping hypothesis, first proposed by Yantis (1992), holds that in the MOT task, observers initially construct a perceptual representation of a virtual polygon at the targets' locations and then continuously update the internal representation of the target configuration along with the motion of the targets. The perceptual grouping thus processes all of the targets as a whole, virtual object in the visual system. Moreover, other studies have provided evidence that observers could form group representations based on spatiotemporal information in MOT task (e.g., changes in motion trajectory [Ogawa \& Yagi, 2002], common motion [Suganuma \& Yokosawa, 2006], or spatial symmetry [Wang et al., 2016]). As to feature-based grouping - that is, when all targets shared one feature token and all distractors shared another - tracking performance would be enhanced because the feature token would bind the targets and segment them from the distractors. On the contrary, when half of the targets and half of the distractors shared one feature token and the remaining objects shared another, tracking performance would be impaired relative to either the "homogeneous" condition, in which all objects had the same features, or a "diversity" condition, in which the targets and distractors all had different features. This was due to the automatic grouping of targets and distractors, even though such grouping was irrelevant to the task instructions and contrary to the task demands (Erlikhman et al., 2013; Wang et al., 2016). Similarly, Howe and Holcombe (2012) demonstrated that, for instance, when the targets were green squares with red centers and the distractors were red squares with green centers, observers' tracking performance was still facilitated, probably because the targets were grouped together and segregated from the distractors.

Previous research has shown effects of objects' physical surface properties on attentive tracking. However, in our daily life, almost all objects that people encounter also contain specific semantic information or conceptual knowledge. The processing of objects thus not only occurs at the perceptual level, but also advances to the higher semantic or conceptual category level. The cognitive resources of human beings are not only attracted by objects' individual surface features, but also largely occupied by the processing of objects' semantic information. Thus, visual object knowledge can be organized into different categories innate in reality (Caramazza \& Shelton, 1998; Mahon \& Caramazza, 2011). In a recent study (Wei, Zhang, Lyu, \& Li, 2016), we used typical and nameable objects to examine the influence of objects' category information on tracking performance. We manipulated the categorical distinction between targets and distractors; specifically, the targets belonged to one category (e.g., animals) and the distractors to another (e.g., furniture). That study showed a consistent categorical-distinction advantage in tracking performance, even when the categorical distinction was narrow. Most importantly, the study demonstrated that the tracking facilitation effect was caused not only by visual distinctiveness between the targets and distractors, but also by their semantic-based categorical difference (Wei et al., 2016). However, the mechanism of this categorical-distinction advantage was still unclear. Why and how does the semantic categorical distinction between targets and distractors in the MIT task facilitate tracking? What are the causes that account for the categorical-distinction advantage in tracking performance? 
In light of the previous research (Erlikhman et al., 2013; Liu, Chen, Liu, \& Fu, 2012; Makovski \& Jiang, 2009a, b; Wang et al., 2016; Wei et al., 2016), we speculated four potential reasons for the tracking enhancement effect. First, any visual distinctiveness between targets and distractors might play a major role in facilitating tracking. Since pictures of line drawings were used as the tracking stimuli in our previous study (Wei et al., 2016), visual distinctiveness was much more evident between targets and distractors belonging to different categories than between those belonging to the same category. Observers thus might mainly distinguish targets and distractors according to their visual distinction, which would aid tracking. To test this assumption, we compared observers' tracking performance for upright and inverted objects, with the targets and distractors being categorically distinct, and found that observers showed better tracking performance for upright than for inverted objects (Wei et al., 2016). Therefore, in addition to visual distinctiveness, semantic category information was also involved in the tracking task and contributed to the tracking benefit. In the present study, we set out to provide more evidence, to build on our previous findings from a different perspective.

The second possible reason is that the categorical distinction might change attentional resources' distribution during tracking, and the tracking enhancement effect would then be due to an attentional distribution strategy. This speculation comes from findings in the MOT task. Pylyshyn (2006) used a probe-dot detection technique and showed that probes on distractors were detected less well than probes either on targets or in the empty space between objects. These results indicated that distractors are subject to a highly localized inhibition in the MOT task when the targets and distractors are identical during tracking. Furthermore, Pylyshyn, Haladjian, King, and Reilly (2008) showed that static distractors and distractors on a different depth plane from the targets were not inhibited, suggesting that the role of inhibition is to keep targets from being confused with the distractors in the MOT task. Distractors that can be distinguished from the targets preattentively and are thus less likely to interfere with tracking will not be inhibited. However, the above finding did not rule out the possibility of target enhancement during tracking. Researchers have used the event-related potential (ERP) technique to measure the amplitudes of the attentionrelated ERP components evoked by probes presented on either targets or distractors (Doran \& Hoffman, 2010; Drew, McCollough, Horowitz, \& Vogel, 2009; Sternshein, Agam, \& Sekuler, 2011). They found evidence that visual attention both enhances targets and suppresses distractors during tracking. In light of these findings, we speculated that the categorical distinction between targets and distractors might alter the attentional distribution during tracking. The distractors thus might be easily distinguished from the targets and might not be inhibited in the categorical-distinction condition. The attentional resources originally required by the inhibition process in that case might be saved and used to enhance the targets. Or, according to the biased-competition model (Desimone \& Duncan, 1995), targets categorically distinct from the distractors might receive a much stronger competitive bias. Therefore, it is possible that targets would receive more attentional resources than distractors in the categoricaldistinction condition than in the homogeneous condition. This attentional-distribution strategy might cause the tracking enhancement effect.

The third potential explanation is based on research about the effect of unique features on attentional tracking (Liu et al., 2012; Makovski \& Jiang, 2009a, b). These researchers found that objects' simple features or identities (e.g., color, numbers of one- or two-digit length, or simple Chinese characters) can be stored in visual working memory, which operates in parallel with motion tracking, and can help observers locate targets lost during tracking. Similarly, the unique semantic identities of objects might be stored in visual working memory in order to contribute to error recovery during tracking. Thus, when visual working memory was disturbed or its working load increased due to the identities of objects changing during tracking, observers' performance might be impaired.

A fourth potential reason is that, similar to the spatiotemporal-based grouping and feature-based grouping in the MOT/MIT task, a category-based grouping mechanism might exist when targets and distractors are categorically distinct. Observers could then group targets together and segregate them from distractors on the basis of their category information. During tracking, they would treat the targets and distractors as separate units. By doing this, they could largely avoid interference from the distractors, facilitating tracking performance.

We conducted six experiments to test the proposed four tracking mechanisms: In Experiment 1 we examined the respective effects on tracking performance of categorical distinction versus visual distinctiveness between the targets and distractors. Experiment 2 tested whether the attentional distribution on targets and distractors was changed by categorical distinctions. In Experiment 3 we investigated the role of visual working memory in tracking facilitation. Experiment 4 tested the category-based grouping hypothesis, and in Experiment 5 we checked whether interference due to the distractors during tracking was decreased in the categorical-distinction condition. At the end, Experiment 6 tested whether categorybased grouping in the MIT task was automatic or effortful.

\section{Experiment 1}

Experiment 1 was designed to replicate the tracking enhancement effect when targets and distractors were categorically distinct that had been indicated by our latest study (Wei 
et al., 2016). More importantly, by using pictures of recombined nonobjects that preserved the original objects' visual information, in Experiment 1 we also tested the degree to which the intercategory facilitation effect was caused by visual distinctiveness between the targets and distractors.

\section{Method}

\section{Participants}

Twelve students (eight female, four male), 19-26 years old (mean age $=21.50, S D=2.71$ ) with normal or corrected-tonormal vision, participated in the experiment. All observers provided informed written consent. The study was approved by the institutional review board (ethics committee) of the School of Psychology at Beijing Normal University. All observers received payment for their time.

\section{Equipment and stimuli}

Equipment The experimental task was controlled by software written in C\#. The stimuli were presented on a Founder 17-in. CRT monitor with a resolution of $1,024 \times 768$ pixels and a refresh rate of $85 \mathrm{~Hz}$. Observers responded by pressing buttons on a keyboard and a mouse.

Stimuli Moving objects were presented in a white, square display that subtended $1,024 \times 768$ pixels $\left(40.96^{\circ} \times 30.72^{\circ}\right)$. A central gray fixation cross subtended $40 \times 40$ pixels $\left(1.6^{\circ} \times\right.$ $\left.1.6^{\circ}\right)$. Sixteen pictures were selected as the stimuli from Cycowicz, Friedman, Rothstein, and Snodgrass's (1997) set of 400 line drawings. Eight of the pictures showed land mammals: a bear, cat, dog, donkey, horse, lion, rabbit, and lamb. The eight remaining pictures showed furniture: a bed, chair, couch, desk, dresser, rocking chair, stool, and bench. Each picture was fit to a square of $60 \times 60$ pixels $\left(2.4^{\circ} \times 2.4^{\circ}\right)$ with an imaginary boundary.

In the visual-distinctiveness-only condition, along the horizontal and longitudinal central axis of symmetry, each object in the pictures was divided into four parts, on average, which were randomly recombined into a new picture. The new pictures contained meaningless nonobjects but had the same basic visual information (perceptual components/elements) as the original objects (see the sample pictures in Fig. 1).

The initial locations of the pictures, moving at random at horizontal and vertical velocities between -5 and +5 pixels per frame, were randomly assigned (Pylyshyn, 2006; Pylyshyn et al., 2008). Each object changed its speed and direction randomly at each frame. A repulsion technique was adopted in order to keep the objects from colliding. The objects bounced off each other when their center-to-center distance was less than 60 pixels. They also avoided the edge of the display area. The maximum speed for an object was $24.1 \%$, and the minimum speed was $3.4 \%$ s.

\section{Design}

The experiment was based on a two-factor within-subjects design. By comparing the objects that represent land mammals and furniture with the recombined nonobjects, which preserved the original objects' basic visual information, we tested the effect of visual distinctiveness on tracking performance. Additionally, the categorical distinction of the targets and distractors was manipulated by creating intercategory, intracategory, or homogeneous displays. In the intercategory condition, the four targets were selected from one category, and the four distractors were selected from another category. In the intracategory condition, the four targets and four distractors were randomly selected from the same category. In the homogeneous condition, the eight objects were all the same object, which was chosen randomly from either land mammals or furniture. The homogeneous condition provided the performance baseline for comparison with the other conditions. Table 1 presents the details of the experimental design. The dependent variable was tracking accuracy, defined as the average proportion of correctly identified targets. Because four of the eight objects were targets, chance accuracy was $50 \%$.

\section{Procedures}

Observers sat approximately $57 \mathrm{~cm}$ away from the monitors, so that each pixel subtended $0.04 \mathrm{deg}$ of visual angle. Instructions for the tracking task were presented on the screen. At the start of each trial, a gray fixation cross and eight pictures were displayed. The observers were encouraged to maintain their fixation on the central cross during tracking. Four of the eight pictures, as the targets, flashed five times in $1 \mathrm{~s}$ for the observers to identify and distinguish them from the distractors. Then all of the pictures began to move randomly, and the movement stopped at a random point within 5-8 s, to prevent observers from simply remembering the last frames of the motion instead of tracking the targets. At the end of
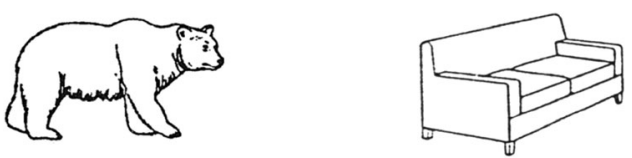

Objects
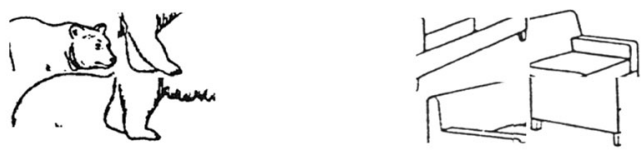

Non-objects

Fig. 1 The sample pictures of objects and nonobjects used in Experiment 1 
motion, the pictures were masked by gray squares subtending $60 \times 60$ pixels $\left(2.4^{\circ} \times 2.4^{\circ}\right)$. The observers had $20 \mathrm{~s}$ to select all four targets with the mouse; the selected squares were highlighted by red frames. Observers pressed the space bar to continue to the next trial (see the sample trial procedure in Fig. 2). Unlike in the traditional MIT task, the observers of the present study were not required to bind the targets' identities and locations together. At the end of the tracking, observers simply reported the locations of the targets.

The experiment began with six practice trials, one for each condition. The object and nonobject conditions were organized into two blocks. Each block consisted of 60 trials (20 trials $\times 3$ categorical distinction levels) displayed in random order. The block order was balanced among observers. Observers rested for at least $1 \mathrm{~min}$ after every 30 trials.

\section{Results and discussion}

First, to test whether the scrambled images of land mammals and furniture had different effects on observers' tracking performance, the tracking accuracies of the three categorydistinction levels under the nonobject condition were separated and calculated for six subconditions: the intercategory scrambled land mammals (the targets being scrambled land mammals and the distractors being scrambled furniture; tracking accuracy: $66.90 \% \pm 14.13 \%$ ), intracategory scrambled land mammals (both the targets and distractors being scrambled land mammals; $63.33 \% \pm 7.71 \%$ ), homogeneous scrambled land mammals (the objects were all the same scrambled land mammal; $66.04 \% \pm 9.44 \%$ ), intercategory furniture (the targets being scrambled furniture and the distractors being scrambled land mammals; $68.75 \% \pm 8.49 \%$ ), intracategory furniture (both the targets and distractors being scrambled furniture; $66.46 \% \pm 10.19 \%$ ), and homogeneous furniture (the objects were all the same scrambled piece of furniture; $64.79 \% \pm 10.47 \%$ ). A two-way repeated measures analysis of variance (ANOVA) revealed that the main effect of the scrambled land mammals/furniture factor was not significant, $F(1$, 11) $=0.404, p=.538, \eta^{2}=.090$; the main effect of the categorical-distinction factor was not significant, $F(2,22)=$
2.830, $p=.081, \eta^{2}=.498$; and the interaction between the two factors was also not significant, $F(2,22)=0.735, p=.491, \eta^{2}$ $=.159$. These results suggested that the scrambled images of land mammals and furniture had no significantly different effects on observers' tracking accuracy. Therefore, we merged the tracking accuracies of scrambled land mammals and furniture by categorical-distinction conditions.

The results of Experiment 1 are presented in Fig. 3. A twoway repeated measures ANOVA revealed a significant main effect of categorical distinction, $F(2,22)=15.389, p<.001, \eta^{2}$ $=.583$. However, the main effect of object/nonobject was not significant, $F(1,11)=0.444, p=.519, \eta^{2}=.039$. The interaction between the two factors was significant, $F(2,22)=$ $11.011, p<.001, \eta^{2}=.500$.

Post-hoc multiple comparisons (with Bonferroni correction) revealed that, in the object condition, the tracking accuracy was significantly better for intercategory distinction than for the intracategory (MD $=0.119, p=.004)$ and homogeneous ( $\mathrm{MD}=0.130, p=.003)$ conditions, which shows that the categorical distinction between targets and distractors significantly promoted tracking performance, suggesting a categorical-distinction advantage. There was no significant difference between the intracategory and homogeneous conditions ( $\mathrm{MD}=0.011, p=1.000$ ). For nonobjects, the tracking accuracies of the three conditions had no significant difference from each other (all $p \mathrm{~s}>.05$ ). This result suggests that when all of objects' categorical information was removed except for visual distinctiveness, observers' tracking performance could not be facilitated.

Simple main-effects analyses revealed that the tracking accuracy was significantly higher for intercategory objects than for intercategory nonobjects, $F(1,11)=9.91, p=.009$. We observed no significant difference between intracategory objects and nonobjects, $F(1,11)=1.02, p=.334$, or between homogeneous objects and nonobjects, $F(1,11)=3.16, p=$ .103 . These results suggest that the visual distinctiveness of targets and distractors was not the reason for the categoricaldistinction advantage in tracking performance. Even though the perceptual processing of objects and nonobjects in the present study was different, their basic lines and visual information were similar. If visual distinctiveness were the main cause of

Table 1 The three categorical distinction conditions of Experiment 1

\begin{tabular}{llc}
\hline Condition & Targets & Distractors \\
\hline Intercategory & $\begin{array}{l}\text { land mammals A, land mammals B, land mammals C, } \\
\text { land mammals D } \\
\text { land mammals A, land mammals B, land mammals C, } \\
\text { land mammals D } \\
\text { land mammals A, land mammals A, land mammals A, } \\
\text { land mammals A }\end{array}$ & $\begin{array}{c}\text { furniture A, furniture B, furniture C, furniture D } \\
\text { land mammals E, land mammals F, land mammals G, } \\
\text { Homogeneous }\end{array}$ \\
\hline
\end{tabular}

A, B $, \ldots, \mathrm{H}$ represent the pictures of land mammals or furniture used as stimuli. The land mammals and furniture targets and distractors were randomly assigned, although only one particular combination is shown here. Please see the text for more details. 


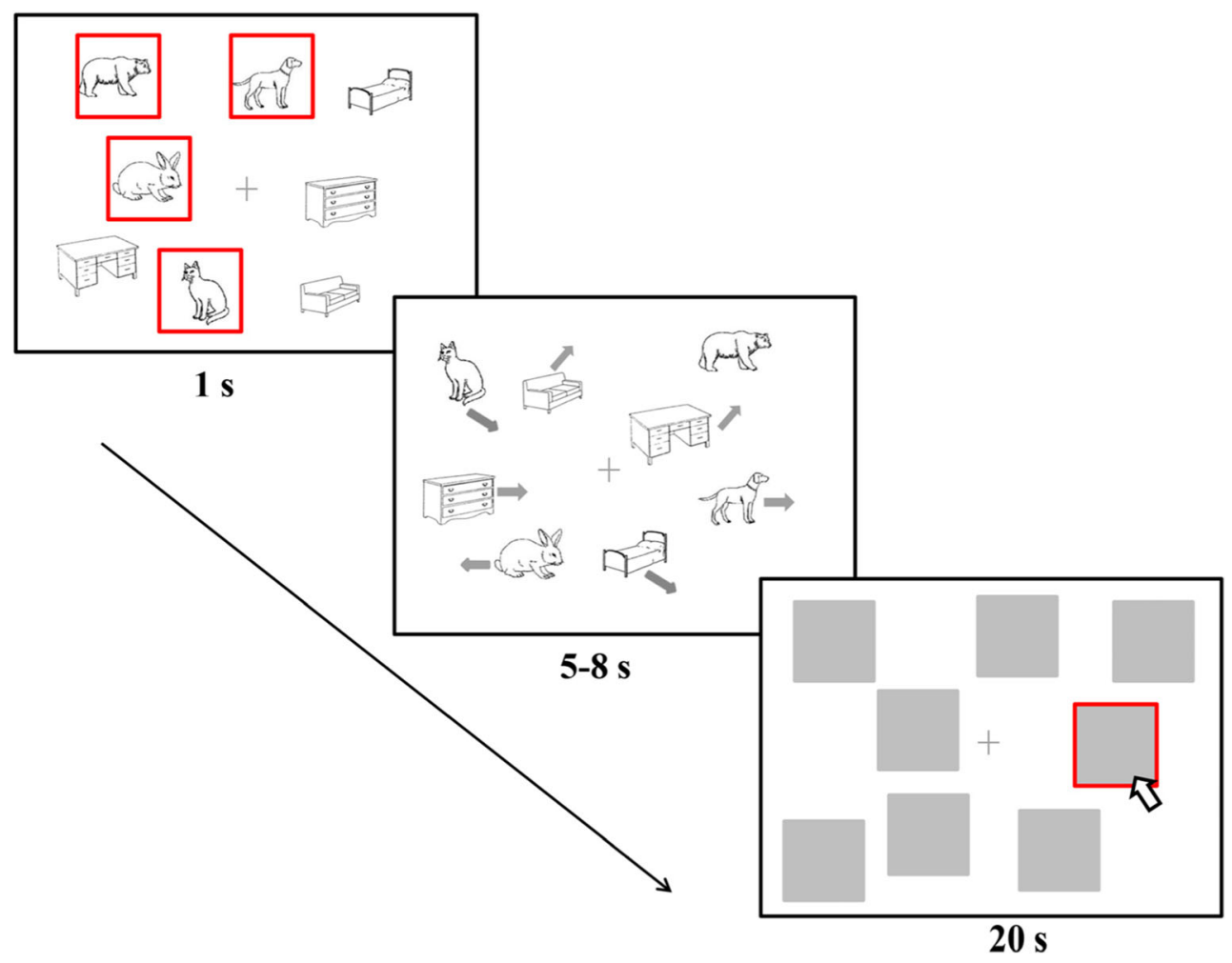

Fig. 2 Sample illustrations of a trial in the intercategory condition of Experiment 1. The land mammals surrounded by red squares were the targets, indicated by flashing five times in $1 \mathrm{~s}$ during the experiment.

the categorical-distinction advantage, the intercategory nonobjects should also have promoted tracking performance relative to the intracategory and homogeneous nonobjects, and the tracking performance for intercategory objects and nonobjects should not have differed significantly. Since the reverse results were demonstrated in Experiment 1,

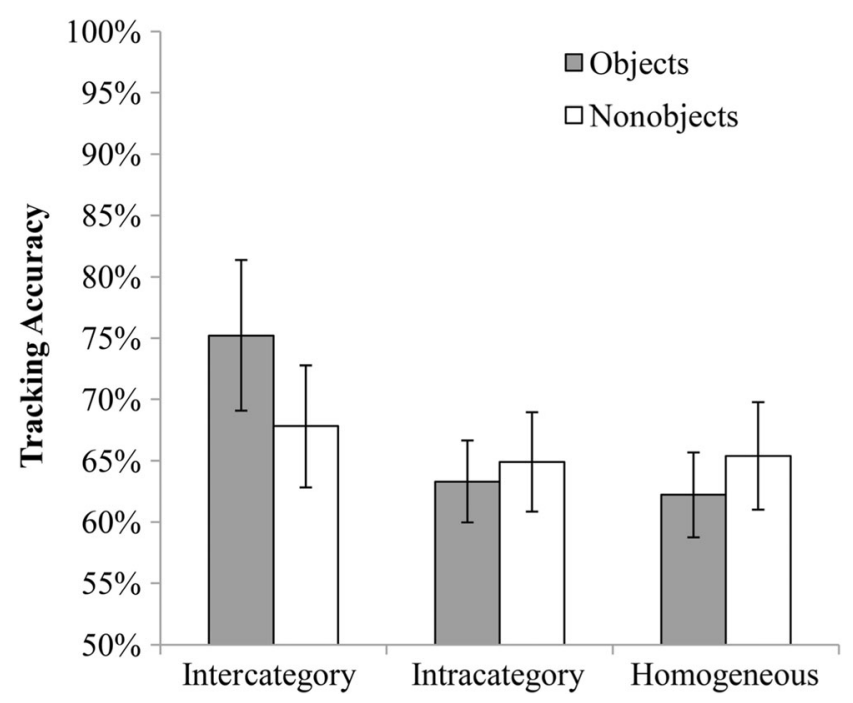

Fig. 3 Tracking accuracy as a function of categorical distinction and objects/nonobjects in Experiment 1 (error bars show \pm 1 standard deviation of the mean)
Observers tracked the targets during the random motion of objects for 5-8 s. After the motion stopped, the objects were masked by gray squares. Observers had to select all targets with a mouse

we inferred that visual distinctiveness itself does not lead to the intercategory facilitation effect.

In our previous study (Wei et al., 2016), we found that the intercategory facilitation effect was reduced for inverted relative to upright objects. The manipulation of inverted images only weakened the objects' semantic category representations, and the tracking enhancement effect still existed in the intercategory condition. However, in the present experiment the nonobjects eliminated all of the category information and left only the visual information, and the intercategory facilitation effect disappeared. These two experiments' results together further indicated that objects' category information does play an important role in facilitating tracking. Even if we could not completely exclude the role of visual distinctiveness in promoting tracking, we did conclude that the main reason for the intercategory facilitation effect was not objects' visual distinctiveness, but their semantic-based categorical information. In the next experiment we explored possible reasons for the intercategory facilitation effect at the attentional level.

\section{Experiment 2}

In Experiment 2 we tested the second hypothesis, that the intercategory facilitation effect might arise from a change in 
attentional strategy. The targets might receive more attention due to the apparent distinction between targets and distractors in the intercategory condition, as compared to the intracategory and homogeneous conditions. We used dual tasks (MIT and a probe detection task) to examine whether the attentional strategy shifted in the intercategory condition. In the dual-task paradigm, observers are required to detect whether a probe has occurred in a particular location while tracking multiple objects at the same time. This task has been used with success to examine the inhibition of distractors in the MOT task (Pylyshyn, 2006; Pylyshyn et al., 2008). At the same time, the performance of probe detection provides an indication of the availability of attentional resources and serves as a measure of either attentional enhancement or inhibition.

\section{Method}

Participants Seventeen students (nine female, eight male), $18-29$ years old (mean age $=23.53, S D=3.68$ ) with normal or corrected-to-normal vision, participated in the experiment. All observers provided informed written consent. The study was approved by the institutional review board (ethics committee) of the School of Psychology at Beijing Normal University. All observers received payment for their time.

Design, equipment, stimuli, and procedures The experiment had a two-factor within-subjects design. We manipulated probe locations and categorical distinction between targets and distractors. As in Experiment 1, categorical distinction also consisted in three conditions: intercategory, intracategory, and homogeneous. In the present and following experiments, only whole objects were used. The probe flashes during tracking were presented on targets or distractors. The probe was a gray square subtending $60 \times 60$ pixels $\left(2.4^{\circ} \times 2.4^{\circ}\right)$ that appeared randomly 2,3 , or $4 \mathrm{~s}$ after the start of the objects' movement in each trial and lasted for $60 \mathrm{~ms}$, to avoid a ceiling effect for detection. Observers were instructed to track the targets and monitor the appearance of the probe simultaneously. In each trial, after reporting the location of each target, observers had to report whether they had seen a gray square appear on the moving objects. If yes, they were to press "Y" on the keyboard; if no, they were to press "N."

In total, 240 trials $(40$ trials $\times 3 \times 2)$ were displayed in a random order. On half of the trials there were no probes. That is, the probes occurred on only $50 \%$ of the trials, to eliminate observers' response bias. Observers rested for at least $1 \mathrm{~min}$ after every 40 trials.

The observers' tracking performance and probe detection scores were measured as dependent variables. All other aspects of the experiment were the same as in Experiment 1.

\section{Results and discussion}

Figure 4 presents the results for tracking accuracy. The tracking accuracy of trials with no probes was used as a baseline for comparison. A two-way repeated measures ANOVA revealed a significant main effect of category distinction, $F(2,32)=$ $74.683, p<.001, \eta^{2}=.824$. However, the main effect of probe location was not significant, $F(2,32)=2.203, p=.137, \eta^{2}=$ .121. The interaction between the two factors was also not significant, $F(4,64)=2.106, p=.102, \eta^{2}=.116$.

Post-hoc tests of category distinction with Bonferroni correction indicated that, consistent with the results of Experiment 1, the tracking accuracy was significantly higher in the intercategory than in the intracategory $(\mathrm{MD}=.178, p<$ .001 ) or homogeneous ( $\mathrm{MD}=.228, p<.001$ ) condition. Intracategory tracking performance was significantly better than homogeneous tracking performance $(\mathrm{MD}=.048, p=$ .001 ). The tracking accuracy results suggest that the tracking task was well controlled, and on this basis, the comparison of probe detection score among different conditions was reliable.

The probe detection results are presented in Fig. 5. A twoway repeated measures ANOVA revealed a significant main effect of probe location, $F(1,16)=16.816, p=.001, \eta^{2}=.512$, which suggested that the targets attracted more attention than the distractors during tracking. This result was consistent with previous research (Pylyshyn, 2006; Pylyshyn et al., 2008). The main effect of category distinction was not significant, $F(2,32)=0.550, p=.582, \eta^{2}=.033$. The interaction between the two factors was also not significant, $F(2,32)=$ $0.165, p=.849, \eta^{2}=.010$.

To further explore the attentional distributions in the three categorical-distinction conditions, we introduced a new index, the probe detection difference between targets and distractors,

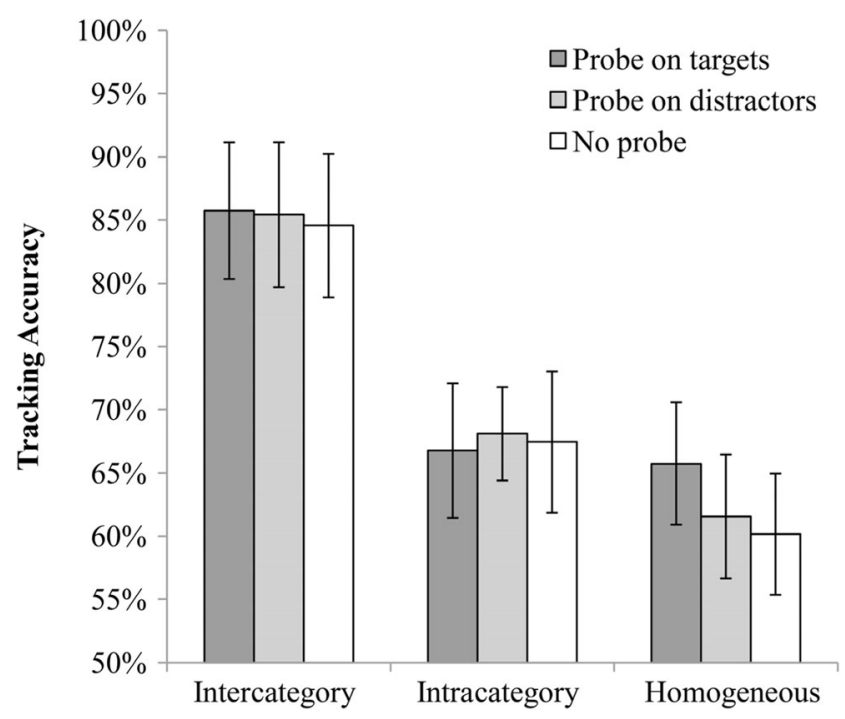

Fig. 4 Tracking accuracy as a function of categorical distinction and probe location in Experiment 2 (error bars show \pm 1 standard deviation of the mean) 
to test whether observers distributed more attentional resources on targets than on distractors in the intercategory distinction condition. There was again no significant difference among the three conditions (probe detection score of targets minus that of distractors: intercategory $\mathrm{MD}=.112$; intracategory $\mathrm{MD}=.153$; homogeneous $\mathrm{MD}=.118$ ).

The finding that the probe detection difference between targets and distractors had no significant difference among the three conditions suggests that the attentional distributions were similar in all the tested conditions. As compared to the distractors, the targets received more attentional resources during tracking in all conditions, and attentional strategy was not shifted by the categorical manipulation. Therefore, the intercategory facilitation effect was not caused by changed attentional distributions. Having excluded the reason of attentional strategy, we examined the role of visual working memory in the intercategory facilitation effect in Experiment 3.

\section{Experiment 3}

In Experiment 3 we examined whether the reason for the intercategory facilitation effect was that the unique identities of targets were stored in visual working memory and assisted the recovery of lost targets. We assessed the categorical distinction advantage under two conditions: fixed objects and changing objects. In the fixed-objects condition, the objects were consistent throughout tracking. In the changing-objects condition, the original objects changed to new objects twice during tracking. If the identities of the targets are stored in visual working memory and the updating of memory is slow

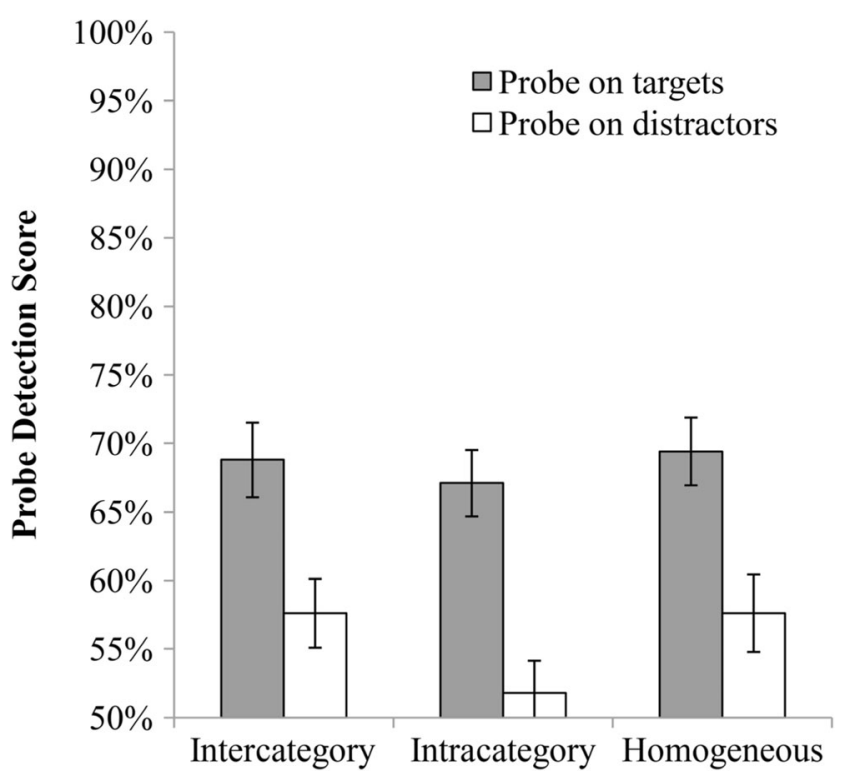

Fig. 5 Probe detection score as a function of categorical distinction and probe location in Experiment 2 (error bars show \pm 1 standard error of the mean) or effortful, the intercategory facilitation effect should be reduced in the changing-objects condition.

\section{Method}

Participants Fifteen students (six female, nine male), 19-27 years old (mean age $=22.93, S D=2.58$ ) with normal or corrected-to-normal vision, participated in the experiment. All observers provided informed written consent. The study was approved by the institutional review board (ethics committee) of the School of Psychology at Beijing Normal University. All observers received payment for their time.

Design, equipment, stimuli, and procedures We manipulated two factors: categorical distinction between the targets and distractors (intercategory, intracategory, and homogeneous) and object consistency. The categorical distinction factor was the same as in Experiments 1 and 2. The difference was that the two categories of objects utilized in Experiment 3 were animals and tools. The object consistency factor included two conditions: fixed objects and changing objects. In the first condition, the objects' identities were preserved during tracking; in the second, the original objects were changed to new objects within the same category at 3 and $5 \mathrm{~s}$ after the start of the movement. The changed objects during tracking would increase the working memory load as a result of the identities updating (Makovski \& Jiang, 2009a, b). To preserve the categorical arrangement among the three categorical distinction conditions after the object change, the animals were always changed to other animals, and tools were changed to other tools.

Sixteen pictures of animals and 16 pictures of tools were also selected from Cycowicz et al.'s (1997) set of 400 line drawings. The 16 animal pictures were of a bear, cat, dog, donkey, horse, lion, rabbit, lamb, camel, deer, elephant, fox, gorilla, pig, squirrel, and hippopotamus. The 16 tool pictures were of an axe, broom, brush, chisel, clothespin, hammer, nail, nail file, nut, pliers, ruler, saw, scissors, screw, screwdriver, and wrench. Each picture was fit to a square of $60 \times 60$ pixels $\left(2.4^{\circ} \times 2.4^{\circ}\right)$ with an imaginary boundary. The moving objects were presented within a white rectangular display that subtended $800 \times 600$ pixels $\left(32^{\circ} \times 24^{\circ}\right)$.

Unlike in Experiments 1 and 2, at the start of each trial in Experiment 3 four of the eight pictures flashed four times in $1.6 \mathrm{~s}$ for observers to identify these targets. After the flashes disappeared, all of the pictures remained still for another $1 \mathrm{~s}$. Then they began to move randomly at a constant speed of $17 \%$ s for a randomly selected duration between 7 and $9 \mathrm{~s}$. All other aspects of the experiment were the same as in Experiments 1 and 2.

The fixed-objects and changing-objects conditions were organized into separate blocks, each consisting of 60 randomly displayed trials (20 trials $\times 3$ categorical distinction levels), 
for 120 trials in total. Half of the observers took the fixedobjects block first, and the other half took the changingobjects block first. Observers rested for at least $1 \mathrm{~min}$ after every 30 trials.

\section{Results and discussion}

The results of Experiment 3 are presented in Fig. 6. A twoway repeated measures ANOVA revealed a significant main effect of category distinction, $F(2,28)=67.875, p<.001, \eta^{2}=$ .829. However, the main effect of object consistency was not significant, $F(1,14)=0.496, p=.493, \eta^{2}=.034$. The interaction between the two factors was significant, $F(2,28)=3.357$, $p=.049, \eta^{2}=.193$.

Post-hoc multiple comparisons (with Bonferroni correction) revealed that, in the fixed conditions, tracking accuracy was significantly better for intercategory than for intracategory $(\mathrm{MD}=.148, p<.001)$ or homogeneous $(\mathrm{MD}=.201, p<.001)$ trials, which was consistent with the results of Experiments 1 and 2, suggesting an intercategory facilitation effect. The tracking performance in the intracategory condition was also significantly better than that in the homogeneous condition ( $\mathrm{MD}=.053, p<.001$ ), which was not consistent with the results of Experiment 1, in which no difference emerged between the intracategory and homogeneous conditions. One possible explanation is that the movement speed of the objects in Experiment 3 was much slower than that in Experiment 1. Observers thus could process and utilize objects' unique identities more fully to aid tracking under the slower speed.

As for the changing conditions, intercategory was significantly better than both intracategory $(\mathrm{MD}=.193, p<.001)$ and homogeneous ( $\mathrm{MD}=.210, p<.001)$ performance. Here, however, we found no significant difference between

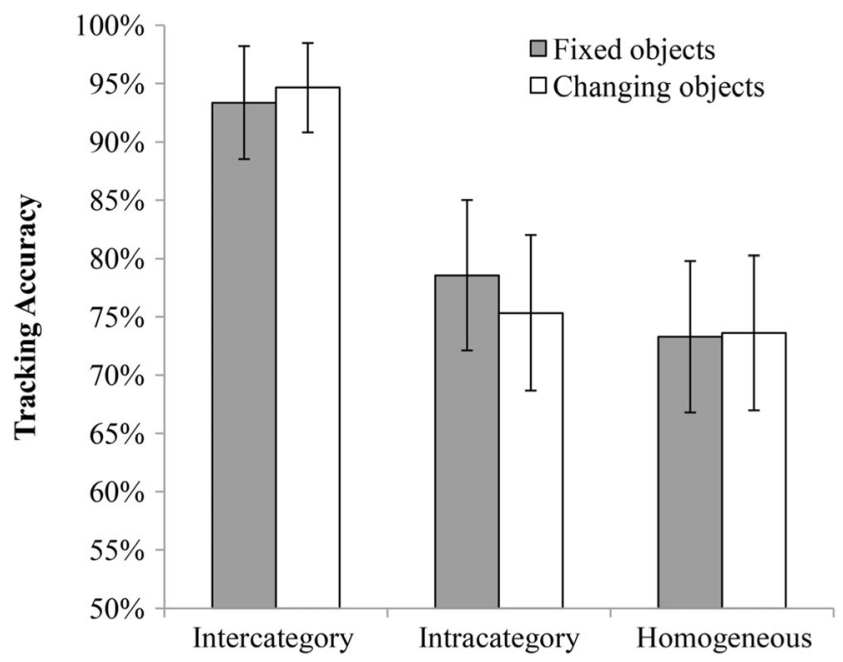

Fig. 6 Tracking accuracy as a function of categorical distinction and object consistency in Experiment 3 (error bars show \pm 1 standard deviation of the mean) intracategory and homogeneous conditions $(\mathrm{MD}=.017, p=$ .829). This result suggested that when objects changed identities twice during tracking, a categorical-distinction advantage in tracking performance still existed.

Simple main-effects analyses revealed that the difference in tracking accuracies between the fixed and changing intracategory conditions approached significance, $F(1,14)=$ $3.89, p=.069$. We observed no significant difference between the fixed and changing intercategory conditions, $F(1,14)=$ $1.48, p=.244$, or the fixed and changing homogeneous conditions, $F(1,14)=0.08, p=.782$.

The results above confirmed that a visual working memory mechanism was not the main cause of the intercategory facilitation effect, for two reasons: One is that if tracking in the intercategory condition were facilitated mainly by searching for and recovering the lost targets according to their unique identities stored in visual working memory, the changing objects would have increased the load of visual working memory and disturb tracking performance. The result that observers' tracking performance in the fixed and changed intercategory conditions had no significant difference provided evidence against such a visual working memory mechanism. The other is that in both the fixed intercategory and fixed intracategory conditions, the eight objects all had unique identities. If the same visual working memory mechanism played a major role in facilitating tracking, similar tracking performances should have been achieved in the two conditions. However, observers' much higher tracking accuracy in the fixed intercategory than that in the fixed intracategory condition suggested that observers adopted different strategies under the two conditions.

However, the results above suggested that, relative to the fixed objects, the changing objects only tended to impair observers' tracking performance in the intracategory condition. This was consistent with the findings of Makovski and Jiang (2009b) that a color change reduced the color uniqueness advantage in tracking performance. The intracategory distinction condition in the present study was similar to their all-unique condition, in which eight objects had eight different colors. Thus, we inferred that in the intracategory condition, observers might encode and store the targets' identities in visual working memory to aid tracking. But in the intercategory condition, observers adopted a different strategy to enhance tracking much more efficiently. In Experiment 4 we tested the semantic category-based grouping hypothesis in this condition.

\section{Experiment 4}

In Experiment 4 we tested the hypothesis of semantic category-based grouping. We proposed that the reason for the tracking facilitation effect in the MIT task was that 
observers organized the targets into one group and segregated them from the distractors on the basis of their categorical difference. From the grouping hypothesis, we conjectured that if the categorical distinction between targets and distractors were removed-and thus the corresponding target grouping disrupted-observers' tracking performance would be disturbed. We manipulated four conditions to examine the influence of disrupted target grouping on tracking performance.

\section{Method}

Participants Nineteen students (ten female, nine male), 1830 years old (mean age $=21.68, S D=3.16$ ) with normal or corrected-to-normal vision, participated in the experiment. Four participants were eliminated because their tracking accuracies were below $50 \%$. All observers provided informed written consent. The study was approved by the institutional review board (ethics committee) of the School of Psychology at Beijing Normal University. All observers received payment for their time.

Design, equipment, stimuli, and procedures This experiment was based on a one-factor within-subjects design. We designated four conditions: objects fixed, objects changing, category reversed, and category mixed. The objects-fixed condition was the same as the intercategory fixed-objects condition in Experiment 3, in which the targets' and distractors' categorical distinction was preserved throughout the trial. The objects-changing condition was the same as the intercategory changing-objects condition in Experiment 3, in which the targets and distractors changed to new objects twice during tracking, with their categorical distinction preserved. The category-reversed condition was the same as the objectschanging condition, except that the targets and distractors swapped their categories every time identities changed. For example, at the start of the movement, the targets were four animals and the distractors were four tools. After the identity changing, the targets were changed to new tools and the distractors to new animals. In the next change, the targets were changed back to new animals and the distractors to new tools. In the category-mixed condition, the targets' and distractors' categorical distinction was eliminated after the change. For example, during the first $3 \mathrm{~s}$ of the movement, the targets were four animals and the distractors were four tools. During the identity changing, both the targets and the distractors were changed to two new animals and two new tools. As a result, the original categorical distinction between targets and distractors was eliminated.

The identity changing always happened 3 and $5 \mathrm{~s}$ after the start of the objects' movement. A total of 80 trials (20 trials $\times$ 4 ) were displayed in random order. All other aspects of the experiment were the same as in Experiment 3.

\section{Results and discussion}

The results of Experiment 4 are shown in Fig. 7. A repeated measures ANOVA indicated a significant main effect, $F(3,42)=29.051, p<.001, \eta^{2}=.675$. Post-hoc tests with Bonferroni correction showed that the tracking accuracies in the objects-fixed and objects-changing conditions had no significant difference $(\mathrm{MD}=.011, p=1.000)$, which was consistent with the results of Experiment 3. The tracking performances of observers in the objects-fixed and objects-changing conditions were both significantly better than performance in the category-reversed and category-mixed conditions (objects fixed vs. category reversed: $\mathrm{MD}=.125, p<.001$; objects fixed vs. category mixed: $\mathrm{MD}=.202, p<.001 ;$ objects changing vs. category reversed: $\mathrm{MD}=.114, p=.001$; objects changing vs. category mixed: $\mathrm{MD}=.191, p<.001)$. The tracking accuracy of observers in the category-reversed condition was marginally significantly better than that in the category-mixed condition $(\mathrm{MD}=$ $.077, p=.053)$. The results above verified the conjecture of our semantic category-based grouping hypothesis. When the categorical distinctions of targets and distractors were exchanged or mixed, and especially when the categorical distinction was removed after the identity changing, tracking performance was significantly impaired. In Experiment 5 we provided more evidence for the category-based grouping hypothesis that organizing the targets and distractors into respective groups effectively reduced the distractors' interference with tracking.

\section{Experiment 5}

In Experiment 5 we investigated why category-based target grouping facilitated tracking. We manipulated the number of distractors and proved that category-based target grouping greatly reduced the distractors' interference with tracking.

\section{Method}

Participants Fifteen students (12 female, three male), 18-26 years old (mean age $=21.27, S D=2.31$ ) with normal or corrected-to-normal vision, participated in the experiment. Three participants were eliminated because their tracking accuracies were below 50\%. All observers provided informed written consent. The study was approved by the institutional review board (ethics committee) of the School of Psychology at Beijing Normal University. All observers received payment for their time.

Design, equipment, stimuli, and procedures The experiment had a two-factor within-subjects design. We manipulated the number of distractors and the categorical distinction 


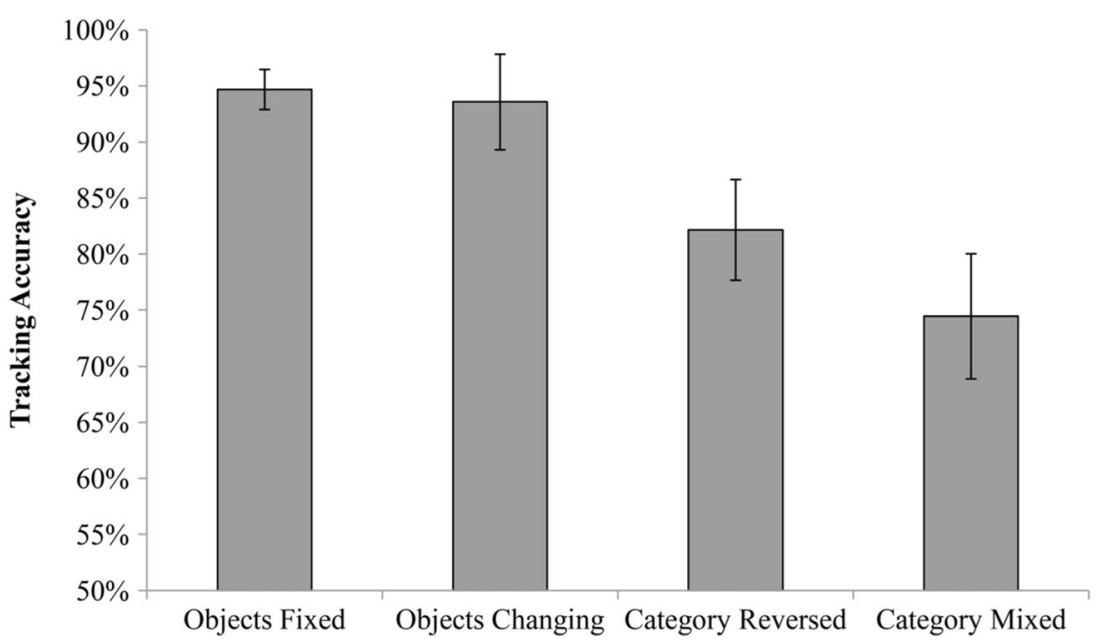

Fig. 7 Tracking accuracy as a function of category condition in Experiment 4 (error bars show \pm 1 standard deviation of the mean)

between targets and distractors. We instructed observers to track four targets among either four, six, or eight distractors. The categorical distinction factor was the same as in Experiments 1, 2, and 3, including three levels: intercategory, intracategory, and homogeneous.

The objects were presented in a white rectangular display that subtended $1,024 \times 768$ pixels $\left(40.96^{\circ} \times 30.72^{\circ}\right)$. Apart from this, the equipment, stimuli, and procedures were the same as in the fixed-objects condition in Experiment 3.

The three levels of distractor number were organized into three blocks, each consisting of 60 trials $(20$ trials $\times 3$ categorical distinction levels) displayed in random order. The block order was balanced among observers. In total, there were 180 trials in the experiment.

\section{Results and discussion}

The results of Experiment 5 are presented in Fig. 8. A two-way repeated measures ANOVA showed that the main effect of distractor number was significant, $F(2,22)=49.430, p<.001$, $\eta^{2}=.818$, as was the main effect of category distinction, $F(2$, 28) $=78.884, p<.001, \eta^{2}=.878$. The two factors' interaction was also significant, $F(4,44)=10.924, p<.001, \eta^{2}=.498$.

The further multiple comparison with Bonferroni correction showed that when four distractors were in the display, the tracking accuracy was significantly higher in the intercategory than in the intracategory (MD $=.145, p<.001)$ and homogeneous (MD $=.178, p<.001$ ) conditions. We found no significant difference between the intracategory and homogeneous conditions ( $\mathrm{MD}=.033, p=.105)$. The same tendencies were revealed in the conditions of six and eight distractors. When there were six distractors, the tracking accuracy of observers in the intercategory condition was significantly higher than the accuracies in the intracategory $(\mathrm{MD}=.190, p<.001)$ and homogeneous $(\mathrm{MD}=.234, p<.001)$ conditions, with no significant accuracy difference between the intracategory and homogeneous conditions ( $\mathrm{MD}=.044, p=.129$ ). When there were eight distractors, the tracking accuracy in the intercategory condition was again significantly higher than those in the intracategory $(\mathrm{MD}=.266, p<.001)$ and homogeneous $(\mathrm{MD}=.299, p<.001)$ conditions, with no significant difference between the tracking accuracies in the intracategory and homogeneous conditions (MD $=.033, p=.285$ ). Consistent with the findings of Experiments 1,2, and 3, when there were four targets and four, six, or eight distractors, the results all revealed an intercategory facilitation effect.

Further multiple comparisons of the three levels of distractor number (with Bonferroni correction) showed that for the intracategory and homogeneous conditions, as the number of distractors increased, tracking performance significantly decreased (intracategory: 4 vs. $6, \mathrm{MD}=.071, p=.003 ; 4$ vs. 8 , $\mathrm{MD}=.160, p<.001 ; 6$ vs. $8, \mathrm{MD}=.089, p=.001$; homogeneous: 4 vs. $6, \mathrm{MD}=.082, p=.004 ; 4$ vs. $8, \mathrm{MD}=$ $.160, p<.001 ; 6$ vs. $8, \mathrm{MD}=.078, p=.009$ ). However, in the intercategory condition, when the number of distractors was increased from four to six and again from six to eight, attentive tracking was not disturbed ( 4 vs. $6, \mathrm{MD}=.026, p=.070 ; 6$ vs. 8 , $\mathrm{MD}=.013, p=.890$ ). Only when the distractor number increased from four to eight was tracking performance significantly impaired ( 4 vs. $8, \mathrm{MD}=.039, p=.037$ ). The results showed that the intercategory distinction could significantly reduce the distractors' interference with tracking. This could be attributed to the grouping of targets according to their categorical difference from the distractors. Observers might treat the targets as one unit, thus segregating the targets better from the distractors and preventing interference of the distractors with tracking.

\section{Experiment 6}

In Experiment 6 we investigated whether or not categorybased grouping is automatic. Previous research had validated 
that when targets and distractors shared the same spatiotemporal properties (e.g., common motion [Suganuma \& Yokosawa, 2006] or spatial symmetry [Wang et al., 2016]) or surface features (such as color, size, shape, combination, or interpolation: Erlikhman et al., 2013; Keane et al., 2011), observers' tracking performance was impaired. This indicated that both spatiotemporal-based and feature-based grouping are automatic in the MOT/MIT task; that is, even when the targetdistractor grouping was contrary to the task demands and had a negative effect on tracking performance, it still could not be resisted. In this experiment, we designed a new condition in which half of the targets and half of the distractors belonged to the same category, and the remainder belonged to another category. If observers' tracking performance in this condition were worse than in the homogeneous condition, we could conclude that category-based grouping was automatic. Otherwise, this grouping mechanism might be goal-directed and effortful.

Method Participants Fourteen students (ten female, four male), 19-28 years old (mean age $=22.36, S D=2.50$ ) with normal or corrected-to-normal vision, participated in the experiment. All observers provided informed written consent. The study was approved by the institutional review board of the School of Psychology at Beijing Normal University. All observers received payment for their time.

Design, equipment, stimuli, and procedures The experiment was based on a one-factor within-subjects design. Besides the intercategory, intracategory, and homogeneous conditions, which were the same as in the object conditions in Experiment 1, we added another condition: mixed-category. In this condition, two targets and two distractors were selected from one category - here, land mammals — while the remaining two targets and two distractors were selected from another category-here, furniture (see Table 2).

The same eight pictures of land mammals and eight pictures of furniture as in Experiment 1 were used as the tracking stimuli. There were always four targets and four distractors in each trial. Apart from this, the equipment, stimuli, and procedures were the same as in the fixed-objects condition in Experiment 3.

In total, 80 trials (20 trials $\times 4$ conditions) were displayed randomly in the experiment, and observers rested for at least $1 \mathrm{~min}$ after completing 40 trials.

\section{Results and discussion}

The results of Experiment 6 are shown in Fig. 9. A repeated measure ANOVA indicated a significant main effect, $F(3,39)$ $=17.068, p<.001, \eta^{2}=.568$. Post-hoc tests with Bonferroni correction showed that observers' tracking accuracy was significantly higher in the intercategory condition than in the other three conditions (intercategory vs. intracategory, $\mathrm{MD}=$ $.085, p=.010$; intercategory vs. homogeneous, $\mathrm{MD}=.114, p$ $=.001$; intercategory vs. mixed-category, $\mathrm{MD}=.117, p<$ .001). Pair-wise comparisons of all the other conditions' tracking accuracies produced no significant differences ( $p s>.05)$. These results suggested that, rather than automatic, semantic category-based grouping is more like a goal-directed and effortful strategy that observers can adopt or ignore according to the specific conditions. Specifically, when a categorical distinction exists between the targets and distractors, observers can utilize that information to form a target grouping and reduce the interference of distractors with tracking.

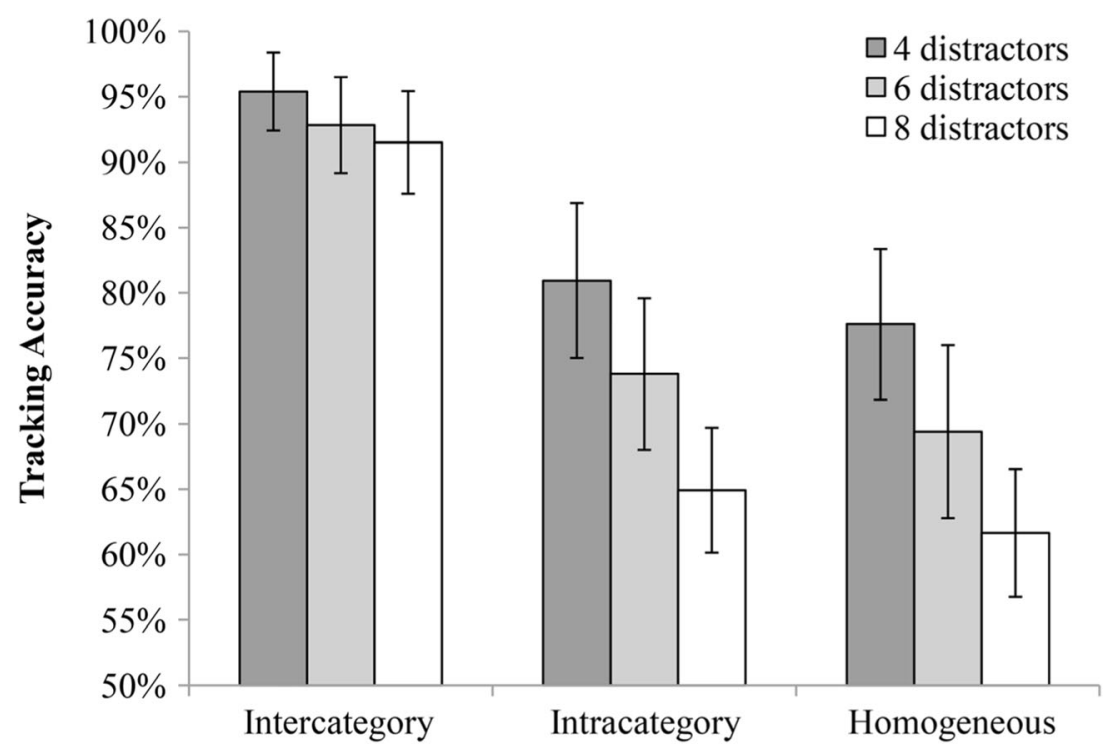

Fig. 8 Tracking accuracy as a function of categorical distinction and number of distractors in Experiment 5 (error bars show \pm 1 standard deviation of the mean) 
Table 2 The four categorical distinction conditions of Experiment 6

\begin{tabular}{|c|c|c|}
\hline Condition & Targets & Distractors \\
\hline Intercategory & $\begin{array}{l}\text { land mammals A, land mammals B, land mammals } \mathrm{C} \text {, } \\
\text { land mammals D }\end{array}$ & furniture $\mathrm{A}$, furniture $\mathrm{B}$, furniture $\mathrm{C}$, furniture $\mathrm{D}$ \\
\hline Intracategory & $\begin{array}{l}\text { land mammals A, land mammals B, land mammals C, } \\
\text { land mammals D }\end{array}$ & $\begin{array}{l}\text { land mammals E, land mammals F, land mammals G, } \\
\text { land mammals H }\end{array}$ \\
\hline Homogeneous & $\begin{array}{l}\text { land mammals A, land mammals A, land mammals A, } \\
\text { land mammals A }\end{array}$ & $\begin{array}{l}\text { land mammals A, land mammals A, land mammals A, } \\
\text { land mammals A }\end{array}$ \\
\hline Mixed-category & land mammals $\mathrm{A}$, land mammals $\mathrm{B}$, furniture $\mathrm{A}$, furniture $\mathrm{B}$, & land mammals $\mathrm{C}$, land mammals $\mathrm{D}$, furniture $\mathrm{C}$, furniture $\mathrm{D}$ \\
\hline
\end{tabular}

$\mathrm{A}, \mathrm{B}, \ldots, \mathrm{H}$ represent the pictures of land mammals or furniture used as stimuli. The land mammals and furniture targets and distractors were randomly assigned, although only one particular combination is shown here. Please see the text for more details.

However, when half the targets and half the distractors belong to the same category and the remainder belong to another category, and category-based target-distractor grouping might impair tracking performance, observers will abandon this grouping strategy in order to avoid its negative effect on tracking.

\section{General discussion and conclusions}

In the present study, we adopted a variant of the MIT task and conducted six experiments to investigate the mechanism behind the phenomenon of categorical distinction between the targets and distractors facilitating tracking (Wei et al., 2016). In the present MIT variant task, the objects carried identity information, but observers only needed to track the targets' locations and not to bind specific identities to changing locations. This MIT variant has widely been used to explore the influence of the surface features or identities of objects on attentive tracking (Erlikhman et al., 2013; Horowitz et al., 2007; Howe \& Holcombe, 2012; Liu et al., 2012; Makovski \& Jiang, 2009a, b; Ren et al., 2009; Wang et al., 2016). The results from Experiment 1 show that when objects' categorical information was removed and visual information was preserved, observers' tracking performance was not facilitated, suggesting that the visual distinctiveness of targets and distractors in the intercategory condition was not the main cause of the categorical-distinction advantage in tracking performance. In Experiment 2, adopting the dual tasks of attentive tracking and probe detection, we confirmed that the facilitation effect was not caused by a shifted attentionaldistribution strategy; instead, the targets received more attentional resources than the distractors in all conditions. Especially in the intercategory condition, in which the targets were different from the distractors in their category information, the targets did not receive a stronger competitive bias than in the intracategory and homogeneous conditions (Desimone \& Duncan, 1995). In Experiment 3 we provided evidence that the intercategory facilitation effect did not mainly exist because the identities of targets were encoded and stored in visual working memory to assist with the recovery from tracking errors. However, the results of Experiment 3 suggested that the improved tracking performance in the intracategory relative to the homogeneous condition could be attributed to the parallel operations of visual working memory for identities and motion tracking, which was consistent to the findings from previous research (Liu et al., 2012; Makovski \& Jiang, 2009b). In Experiment 4 we found that when the categorical distinction between targets and distractors was reversed or disrupted during tracking, observers' tracking performance was significantly impaired. These results were consistent with the prediction of the semantic category-based grouping hypothesis (Wei et al., 2016). In Experiment 5 we revealed that categorical distinction of the targets and distractors significantly reduced the distractors' interference with tracking, further confirming the grouping hypothesis. Finally, the results from Experiment 6 indicated that category-based grouping is not automatic; on the contrary, it is a goal-directed and effortful strategy. When they realize that the category-based target grouping can facilitate tracking, observers will intentionally adopt this strategy; in contrast, when they notice that category-based target-distractor grouping may interrupt tracking, they will abandon this strategy.

\section{The mechanism of semantic category-based grouping}

The grouping hypothesis originally proposed by Yantis (1992) assumes that in the MOT task, observers initially construct a perceptual representation of a virtual polygon during target designation and then continuously update this internal model with the motion of the targets. Grouping thus consists of two distinct processes: The initial formation of a perceptual group is relatively stimulus-driven and automatic, whereas group maintenance during tracking is goal-directed and effortful (Yantis, 1992). Since the surface properties of moving objects are identical in a typical MOT task, the grouping process is largely based on their spatiotemporal information (e.g., changes in motion trajectory [Ogawa \& Yagi, 2002], common motion [Suganuma \& Yokosawa, 2006], or spatial symmetry [Wang, et al., 2016]), whereas the MIT task, in which the 


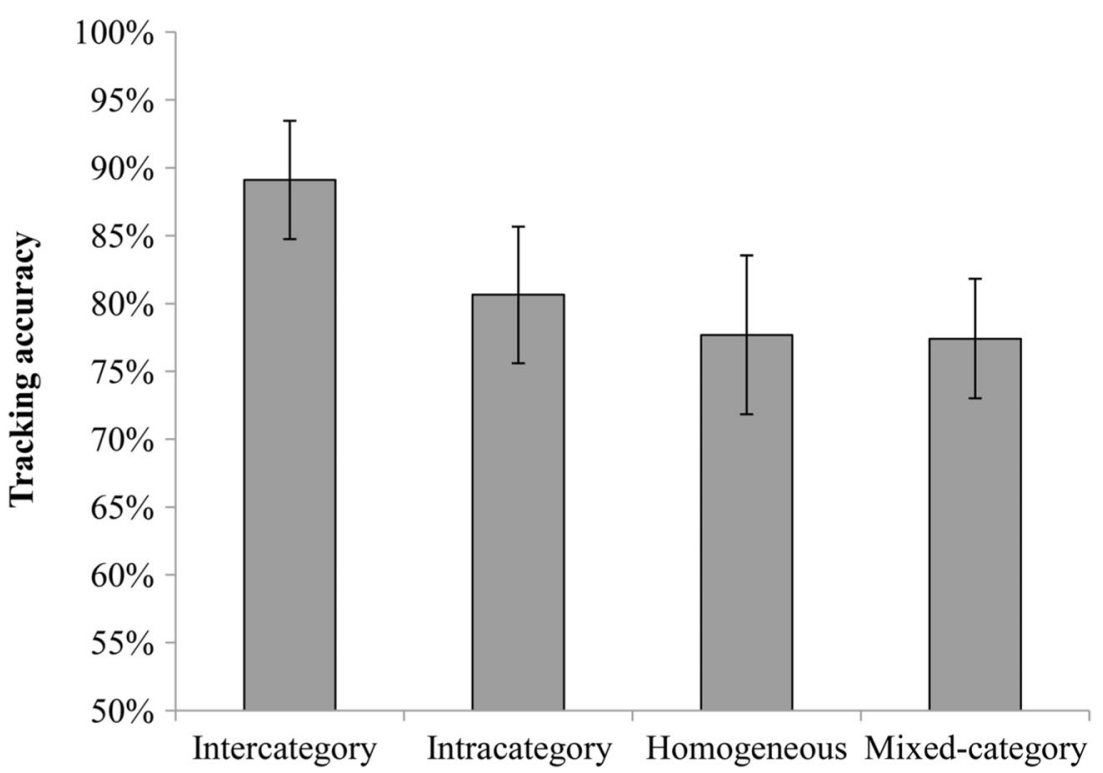

Fig. 9 Tracking accuracy as a function of categorical distinction in Experiment 6 (error bars show \pm 1 standard deviation of the mean)

objects have distinct features, elicits an automatic featurebased grouping effect (Erlikhman et al., 2013; Keane et al., 2011). Observers automatically employ objects' features to create "collections of related objects" to enhance or impair the attentive tracking of multiple objects. In the present study, we provided evidence that observers could use objects' categorical information to form a target grouping and aid tracking. By doing this, they could greatly avoid the distractors' interference with tracking.

Previous studies have validated that identity processing for the objects is involuntary in the MIT variant task (Cohen, Pinto, Howe, \& Horowitz, 2011; Horowitz et al., 2007; Liu et al., 2012; Ren et al., 2009). Specifically, the objects' identities will always be processed, even when they are irrelevant to the task, and the processing consumes extra resources and impairs tracking performance. From the present study, we further inferred that objects' semantic category information could also be accessed during tracking, even at an implicit level. In short, a semantic category-based grouping mechanism exists: In the case of the targets and distractors being categorically distinct, observers can use the category information to distinguish targets from distractors. This raised the questions, how does category-based target grouping facilitate location tracking? and what is the interaction between the category-based grouping and location tracking?

In light of the present findings, we assume that semantic category-based grouping operates parallel to trajectory monitoring (i.e., location tracking). Since category-based grouping is not automatic but goal-directed and effortful, it is more like a flexible strategy available during location tracking. When the targets and distractors are categorically distinct, target grouping can facilitate location tracking, so the strategy will be employed intentionally. However, when half of the targets and half of the distractors belong to the same category and the remainder belong to another category, target-distractor grouping could interrupt tracking, so it will be abandoned. The target-grouping facilitation effect may operate mainly by two means: One is that target grouping can segregate the sets of targets from the distractors and reduce the distractors' interference with tracking (according to the results of Exp. 5). The other may be an error recovery strategy; specifically, when one or more targets are lost during tracking, observers might use their categorical information to find them again. This error recovery strategy is different from the mechanism presented by both Horowitz et al. (2007) and Makovski and Jiang (2009b). In their proposals, if observers lose track of one or more targets, they can search for the targets on the basis of their surface features or of identities stored in visual working memory, and thus continue to track them. The error recovery in the present target-grouping mechanism is not based on the specific identities of targets, but rather on their category information (i.e., their group label). Fundamentally, the error recovery strategy helps observers maintain the targets' grouped representation, to ensure that the targets are treated as a unit.

It should also be noted that monitoring of the targets' trajectories during the motion of multiple objects was necessary here. It was impossible for observers to satisfy the task demands simply through visual search, even in the intercategory condition. There are mainly two reasons: One is that because the objects' motion stopped at a random point of time, observers could not predict when the objects would stop. The unpredictable length of the trial ensured that observers were actively tracking rather than simply searching for and locating targets on the basis of their category information in the last frame of motion. The other reason is that in the present study, the objects moved at a relatively high speed and within a broad 
area, and the high speed of motion did not allow attention to be switched between individual objects. If observers only visually searched for the targets' locations from time to time instead of tracking them during the motion phase, performance would have been much worse than in the present results (Pylyshyn, 2009; Pylyshyn \& Storm, 1988). Post-hoc interviews with some participants about the strategies they had used in the task also showed that it was very difficult to locate the targets accurately using only visual search in the motion of multiple objects.

\section{The similarities and differences between semantic category-based grouping and feature-based grouping}

In MIT variant tasks, whether based on semantic categorybased or feature-based grouping, what the tasks have in common is that target grouping can segregate the targets from distractors, avoid the swapping of targets and distractors, and generally aid tracking. However, the strategies differ in that the former is a goal-directed strategy, whereas the latter is automatic (Erlikhman et al., 2013; Keane et al., 2011). That is, in a feature-based target-distractor grouping condition, observers cannot resist creating "collections of targetsdistractors" and having their performance impaired (Erlikhman et al., 2013), whereas in a category-based targetdistractor grouping condition, observers are able to avoid the adverse effect and achieve the same tracking performance as in the homogeneous condition. This diversity may be due to the different processing loads required by surface features and semantic categories. The processing of surface features requires fewer resources, works automatically, and stays at a low level, whereas the processing of semantic categories is more resource-intensive, is not automatic, and stays at a high level. As a result, observers cannot ignore the objects' surface features but can consciously neglect their category information during location tracking.

However, with regard to the relationship between categorybased and feature-based grouping, some meaningful but still unknown issues need further investigation. One is whether category-based and feature-based forms of grouping are additive. For example, when target grouping based on both surface-feature and semantic-category information is possible, would the tracking facilitation effect be larger than when the target grouping must be based on either surface-feature or category information? Another question is, when objects' surface properties and semantic categories conflict, are observers more likely to organize, for example, objects of the same color or objects of the same category into one group? Exploring these questions will be helpful in clarifying the relationship between feature-based grouping and category-based grouping in the MIT task.

Although the present study has revealed that category-based target grouping is the main mechanism of the intercategory facilitation effect, this conclusion cannot totally exclude the involvement of perceptual distinction, attentional-resource distributions, and visual working memory in the MIT task. These cognitive processes do not play a leading role in conditions in which the targets and distractors are categorically distinct, but they may function in a weak way, and their effect may be detectable under specified conditions.

In the present MIT task, in which objects carried semantic category information, identity processing does not stay at the perceptual level, but advances to the processing of conceptual knowledge and conceptual category, at the knowledge representation level. As we know, people experience the world in terms of categories (Caramazza \& Shelton, 1998; Mahon \& Caramazza, 2011). That is, people tend to remember objects' categorical information but not their specific details, and use the semantic or conceptual category to mainly construct their declarative knowledge. The categorical organization of knowledge, in turn, strongly influences the way that people encode and remember their experiences. The present findings provide evidence that this categorical organization principle also applies in the concurrent processing of multiple objects in dynamic scenes, and that semantic similarity or category can lead to the grouping of randomly moving objects.

Author note This study was supported by the General Program of National Natural Science Foundation of China (Grant No. 31271083) and by the Key Program of National Natural Science Foundation of China (Grant No. 61632014).

\section{References}

Caramazza, A., \& Shelton, J. R. (1998). Domain-specific knowledge systems in the brain: The animate-inanimate distinction. Journal of Cognitive Neuroscience, 10, 1-34.

Cohen, M. A., Pinto, Y., Howe, P. D. L., \& Horowitz, T. S. (2011). The what-where tradeoff in multiple identity tracking. Attention, Perception \& Psychophysics, 73, 1422-1434.

Cycowicz, Y. M., Friedman, D., Rothstein, M., \& Snodgrass, J. G. (1997). Picture naming by young children: Norms for name agreement, familiarity, and visual complexity. Journal of Experimental Child Psychology, 65, 171-237.

Desimone, R., \& Duncan, J. (1995). Neural mechanisms of selective visual attention. Annual Review of Neuroscience, 18, 193-222.

Doran, M. M., \& Hoffman, J. E. (2010). The role of visual attention in multiple object tracking: Evidence from ERPs. Attention, Perception, \& Psychophysics, 72, 33-52. doi:https://doi.org/10. 3758/APP.72.1.33

Drew, T., McCollough, A., Horowitz, T., \& Vogel, E. (2009). Attentional enhancement during multiple-object tracking. Psychonomic Bulletin \& Review, 16, 411-417. doi:https://doi.org/10.3758/PBR.16.3.411

Erlikhman, G., Keane, B., Mettler, E., Horowitz, T., \& Kellman, P. (2013). Automatic feature-based grouping during multiple object tracking. Journal of Experimental Psychology: Human Perception and Performance, 39, 1625-1637. 
Feria, C. (2012). The effects of distractors in multiple object tracking are modulated by the similarity of distractor and target features. Perception, 41, 287-304.

Fougnie, D., \& Marois, R. (2006). Distinct capacity limits for attention and working memory. Psychological Science, 17, 526-534.

Horowitz, T. S., Klieger, S. B., Fencsik, D. E., Yang, K. K., Alvarez, G. A., \& Wolfe, J. M. (2007). Tracking unique objects. Perception \& Psychophysics, 69, 172-184. doi:https://doi.org/10.3758/BF03193740

Howe, P., \& Holcombe A. (2012). The effect of visual distinctiveness on multiple object tracking performance. Frontiers in Perception Science, 3, 307.

Keane, B. P., Mettler, E., Tsoi, V., \& Kellman, P. J. (2011). Attentional signatures of perception: Multiple object tracking reveals the automaticity of contour interpolation. Journal of Experimental Psychology: Human Perception and Performance, 37, 685- 698.

Liu, T. W., Chen, W. F., Liu, C. H., \& Fu, X. L. (2012). Benefits and costs of uniqueness in multiple object tracking: The role of object complexity. Vision Research, 66, 31-38.

Mahon, B. Z., \& Caramazza, A. (2011). What drives the organization of object knowledge in the brain? Trends in Cognitive Sciences, 15, $97-103$.

Makovski, T., \& Jiang, Y. V. (2009a). Feature binding in attentive tracking of distinct objects. Visual Cognition, 17, 180-194.

Makovski, T., \& Jiang, Y. V. (2009b). The role of visual working memory in attentive tracking of unique objects. Journal of Experimental Psychology: Human Perception and Performance, 35, 1687-1697.

Ogawa, H., \& Yagi, A. (2002). The effects of the information of untracked objects on multiple object tracking. Japanese Journal of Psychonomic Science, 21, 49-50.

Oksama, L., \& Hyönä, J. (2004). Is multiple object tracking carried out automatically by an early vision mechanism independent of higherorder cognition? An individual difference approach. Visual Cognition, $11,631-671$.
Oksama, L., \& Hyönä, J. (2008). Dynamic binding of identity and location information: A serial model of multiple-identity tracking. Cognitive Psychology, 65, 237-283.

Pylyshyn, Z., Haladjian, H., King, C., \& Reilly, J. (2008). Selective nontarget inhibition in multiple object tracking (MOT). Visual Cognition, 16, 1011-1021.

Pylyshyn, Z. W. (2006). Some puzzling findings in multiple object tracking: II. Inhibition of moving nontargets. Visual Cognition, 14, 175198.

Pylyshyn, Z. W. (2009). Perception, representation and the world: The FINST that binds. In D. Dedrick \& L. Trick (Eds.), Computation, cognition, and Pylyshyn. Cambridge: MIT Press.

Pylyshyn, Z. W., \& Storm, R. W. (1988).Tracking multiple independent targets: Evidence for a parallel tracking mechanism. Spatial Vision, 3, 179-197.

Ren, D., Chen, W., Liu, C. H., \& Fu, X. (2009). Identity processing in multiple-face tracking. Journal of Vision, 9(5), 1-15. doi:https://doi. org/10.1167/9.5.18

Sternshein, H., Agam, Y., \& Sekuler, R. (2011). EEG correlates of attentional load during multiple object tracking. PLoS ONE, 6, e22660.

Suganuma, M., \& Yokosawa, K. (2006). Grouping and trajectory storage in multiple object tracking: Impairments due to common item motions. Perception, 35, 483-495.

Wang, C., Zhang, X., Li, Y., \& Lyu, C. (2016). Additivity of feature-based and symmetry-based grouping effects in multiple object tracking. Frontiers in Psychology, 7, 657. doi:https://doi.org/10.3389/fpsyg. 2016.00657

Wei, L., Zhang, X., Lyu, C., \& Li, Z. (2016). The categorical distinction between targets and distractors facilitates tracking in Multiple Identity Tracking task. Frontiers in Psychology, 7, 589. doi:https:// doi.org/10.3389/fpsyg.2016.00589

Yantis, S. (1992). Multielement visual tracking: Attention and perceptual organization. Cognitive Psychology, 24, 295-340. 